\title{
Hexamethylene Bisacetamide
}

National Cancer Institute

\section{Source}

National Cancer Institute. Hexamethylene Bisacetamide. NCI Thesaurus. Code 61125.

A hybrid polar-planar compound with potential antineoplastic activity that induces

terminal differentiation, inhibits cell growth, and causes apoptosis in several tumor cell lines. Its precise mechanism of action is unknown. ( $\mathrm{NCI04)}$ 